\title{
Diabetic cardiomyopathy in Zucker diabetic fatty rats: the forgotten right ventricle
}

\author{
Charissa E van den Brom*1,2, Joanna WAM Bosmans 1,2, Ronald Vlasblom¹,2, Louis M Handoko2,3, Marc C Huisman4, \\ Mark Lubberink ${ }^{4}$, Carla FM Molthoff4, Adriaan A Lammertsma ${ }^{4}$, Margriet D Ouwens 6 , Michaela Diamant ${ }^{1}$ and \\ Christa Boer ${ }^{5}$
}

\begin{abstract}
Background: In patients with myocardial infarction or heart failure, right ventricular (RV) dysfunction is associated with death, shock and arrhythmias. In patients with type 2 diabetes mellitus, structural and functional alterations of the left ventricle (LV) are highly prevalent, however, little is known about the impact of diabetes on RV characteristics. The purpose of the present study was to investigate whether $L V$ changes are paralleled by RV alterations in a rat model of diabetes.

Methods: Zucker diabetic fatty (ZDF) and control (ZL) rats underwent echocardiography and positron emission tomography (PET) scanning using [18F]-2-fluoro-2-deoxy-D-glucose under hyperinsulinaemic euglycaemic clamp conditions. Glucose, insulin, triglycerides and fatty acids were assessed from trunk blood. Another group of rats received an insulin or saline injection to study RV insulin signaling.
\end{abstract}

Results: ZDF rats developed hyperglycaemia, hyperinsulinaemia and dyslipidaemia (all $p<0.05$ ). Echocardiography revealed depressed LV fractional shortening and tricuspid annular plane systolic excursion (TAPSE) in ZDF vs. ZL rats (both $p<0.05$ ). A decrease in LV and RV insulin-mediated glucose utilisation was found in ZDF vs. ZL rats (both $p<$ 0.05). LV associated with RV with respect to systolic function $(r=0.86, p<0.05)$ and glucose utilisation $(r=0.74, p<$ 0.05). TAPSE associated with RV MRglu $(r=0.92, p<0.05)$ and $M$-value $(r=0.91, p<0.0001)$ and RV MRglu associated with $M$-value $(r=0.77, p<0.05)$. Finally, reduced RV insulin-stimulated phosphorylation of Akt was found in ZDF vs. ZL $(p<0.05)$.

Conclusions: LV changes were paralleled by RV alterations in insulin-stimulated glucose utilisation and RV systolic function in a rat model of diabetes, which may be attributed to ventricular interdependence as well as to the uniform effect of diabetes. Since diabetic patients are prone to develop diabetic cardiomyopathy and myocardial ischaemia, it might be suggested that RV dysfunction plays a central role in cardiac abnormalities in this population.

\section{Background}

Although been underestimated in the past, the contribution of right ventricular (RV) function to overall myocardial contractility is considerable. RV dysfunction is relevant in a variety of disease states, like pulmonary hypertension, coronary heart disease and heart failure. $\mathrm{RV}$ dysfunction is an independent predictor of outcome

* Correspondence: c.vandenbrom@vumc.nl

1 Department of Internal Medicine/Diabetes Centre, VU University Medical

Centre, Amsterdam, the Netherlands

Full list of author information is available at the end of the article in patients with myocardial infarction [1] and heart failure $[2,3]$. Patients with type 2 diabetes are at increased risk of myocardial infarction [4], however, not much is known on RV involvement in these patients.

While left ventricular (LV) impairment is a common complication of type 2 diabetes mellitus (T2DM), the number of studies focusing on the impact of T2DM on RV function is limited and conflicting [5-8]. We [9] and others $[10,11]$ have shown that alterations in LV energy metabolism, resulting from changes in substrate supply 
and utilisation, contribute to the development of myocardial dysfunction, but this association has not been established for the RV. Despite the association of RV dysfunction with aggravation of myocardial function and outcome in distinct cardiac diseases, its role in diabetic cardiomyopathy is unknown. The purpose of the present study was therefore to investigate whether LV changes are paralleled by RV alterations with respect to glucose utilisation and function in an experimental rat model of diabetes.

\section{Material and methods}

\section{Animals}

All experiments were approved by the Animal Care and Use Committee of the VU University, and were conducted following the European Convention for the Protection of Vertebrate Animals used for Experimental and Other Scientific Purposes, and the Dutch Animal Experimentation Act.

Male Zucker diabetic fatty (ZDF) rats (fa/fa; $\mathrm{n}=12$, Charles River Laboratories Brussels, Belgium) and agematched Zucker lean control rats $(\mathrm{ZL} ;+/+; \mathrm{n}=10)$ underwent echocardiography and positron emission tomography (PET) at 14 weeks of age. Trunk blood was collected for determinations of blood glucose, plasma insulin, fatty acid and triglyceride levels [12]. Hearts were removed and either snap-frozen in dry-ice-chilled isopentane or fixed in $4 \%$ formalin for further biochemical and histological analysis.

\section{Echocardiography}

Echocardiographic analyses were performed as previously described $[9,12]$. Measured parameters for RV function were Doppler-derived cardiac output and tricuspid annular plane systolic excursion (TAPSE). Parameters for RV remodeling and pulmonary vascular remodeling were measured as RV end-diastolic diameter and pulmonary artery acceleration time normalised for cycle length $(\mathrm{PAAT} / \mathrm{cl})$, respectively [13]. Further, estimated RV systolic pressure (eRVSP) was determined by $142^{*} \mathrm{e}^{\left(-11^{*}[\text { PAAT/ }\right.}$ cl]) $[12]$.

\section{Positron emission tomography}

LV and RV glucose metabolism were measured using [18F]-2-fluoro-2-deoxy-D-glucose (18FDG) PET under hyperinsulinaemic euglycaemic clamp conditions, a goldstandard estimate of whole-body insulin sensitivity [9]. Quantification was achieved with compartment analysis, based on a two-tissue compartment model [9].

\section{Histology}

Sections of LV and RV were stained with hematoxylineosin for determination of cardiomyocyte cross-sectional area [12]. Picrosirius red staining was used for analysis of myocardial fibrosis. LV and RV fibrosis were expressed as the percentage tissue area positive for collagen, measured over minimally three randomly chosen areas per ventricle [13].

\section{Western blotting}

A separate group of rats ( $\mathrm{n}=4$, ZDF rats; $\mathrm{n}=4$, ZL rats) received insulin stimulation (10 U.kg-1 BW insulin) or saline as control ( $\mathrm{n}=4$, ZDF rats; $\mathrm{n}=4$, ZL rats) to study RV insulin signaling $[9,14]$ using the following primary antibodies: phospho-Akt-Ser473, phospho-GSK3 $\beta$-Ser9, GSK3 $\beta$ (all Cell signaling Technology, Beverly, MA) and Akt2 (Upstate, Lake Placid, NY).

RV substrate metabolism was studied using glucose transporter 4 (GLUT4; Abcam, Cambridge, MA), phospho-AMP activated protein kinase (AMPK)-Thr172, AMPK (both Cell signaling Technology, Beverly, MA) and pyruvate dehydrogenase kinase-4 (PDK4; Santa Cruz Biotechnology, Santa Cruz, CA) [8]. All signals were normalised to actin (Sigma, Saint Louis, Missouri) or total protein expression.

\section{Statistical analysis}

All data are presented as mean \pm SEM. Group comparisons were performed using student t-test or two-way ANOVA with Bonferroni post-hoc analysis. Correlations were calculated by the Pearson's test. $\mathrm{p}<0.05$ was considered as statistically significant.

\section{Results}

Consistent with a diabetic phenotype, ZDF rats were obese, hyperglycaemic, hyperinsulinaemic and dyslipidaemic compared with ZL rats as shown in Table 1. The clamp-measured $M$-value was significantly lower in ZDF rats than in control rats (Table 1).

Echocardiography in ZDF rats showed a significant decreased in RV and LV systolic function compared to controls (Figure 1A and 1B), as measured by TAPSE and fractional shortening, respectively. Cardiac output remained unchanged in ZDF rats versus controls $(96 \pm 8$ $\mathrm{ml} / \mathrm{min}$ vs. $93 \pm 6 \mathrm{ml} / \mathrm{min}$ for ZDF and ZL rats, respectively, n.s.). TAPSE associated positively with LV fractional shortening $(r=0.86, p<0.05$, Figure $1 \mathrm{E})$. Interestingly, RV diastolic diameter was significantly increased, whereas LV diastolic diameter remained unchanged (Figure 2A). Furthermore, PAAT/cl showed no difference between the two experimental groups $(0.17$ \pm 0.01 for ZDF rats vs. $0.18 \pm 0.01$ for ZL rats, n.s.). Estimated RVSP remained also unchanged between both groups $(21.2 \pm 1.9 \mathrm{mmHg}$ for ZDF rats vs. $23.6 \pm 2.1$ $\mathrm{mmHg}$ for $\mathrm{ZL}$ rats, n.s.). 


\begin{tabular}{lll}
\hline & ZL & ZDF \\
\hline Body weight at killing [g] & $335 \pm 4$ & $355 \pm 8^{*}$ \\
Non-fasting blood glucose [mmol.L-1] & $5.0 \pm 0.1$ & $20.9 \pm 0.8^{*}$ \\
Fasting insulin [pmol.:-1] & $116.9 \pm 11.9$ & $330.7 \pm 69.6^{*}$ \\
Fasting triglycerides [mmol.L-1] & $0.36 \pm 0.02$ & $1.41 \pm 0.20^{*}$ \\
Fasting fatty acids [mmol.L-1] & $0.16 \pm 0.03$ & $0.38 \pm 0.08^{*}$ \\
$M$-value [mg.kg.min-1] & $22.4 \pm 1.0$ & $8.9 \pm 0.8^{*}$ \\
\hline
\end{tabular}

Data are expressed as mean $\pm S E M, n=8,{ }^{*} p<0.05$ vs. ZL

ZL, Zucker lean; ZDF, Zucker diabetic fatty

RV and LV metabolic rates of glucose utilisation (MRglu) measured under hyperinsulinaemic euglycaemic conditions were decreased by $66 \%$ and $60 \%$, respectively, in ZDF versus ZL rats (Figure $1 C$ and $1 D$ ). RV MRglu was positively associated with LV MRglu $(\mathrm{r}=0.74, \mathrm{p}<0.05$, Figure 1F).

TAPSE associated significantly with RV MRglu ( $\mathrm{r}=$ $0.92, \mathrm{p}<0.05)$ and $M$-value $(\mathrm{r}=0.91, \mathrm{p}<0.0001)$ and RV MRglu associated significantly with $M$-value $(\mathrm{r}=0.77, \mathrm{p}<$ 0.05).

$\mathrm{RV}$ weight, $\mathrm{RV} /(\mathrm{LV}$ + septum) ratio, $\mathrm{LV}$ weight and lung weight were similar in ZDF and ZL rats (data not shown). Cross-sectional areas of individual RV cardiomyocytes were similar between both groups, whereas cross sectionals areas of individual LV cardiomyocytes were significantly increased (Figure 2B) in ZDF rats, suggesting different remodeling between ventricles. Further, RV and LV fibrosis remained unchanged between both groups (Figure 2C).

Phosphorylation of Akt and GSK3 $\beta$ were studied to determine RV insulin sensitivity. ZDF and ZL hearts showed similar basal Akt and GSK $\beta$ phosphorylation. RV insulin-dependent phosphorylation of Akt-Ser473 was decreased in ZDF rats (two-way ANOVA interaction $\mathrm{p}=$ 0.05 , Figure $3 \mathrm{~A}$ and $3 \mathrm{C}$ ). Further, insulin injection increased GSK3 $\beta$ phosphorylation significantly in ZL rats, but this increase was blunted in ZDF rats (Figure $3 \mathrm{~B}$ and 3D).

Glucose transporter 4 (GLUT4) and AMP-activated kinase (AMPK) protein expression was decreased in ZDF rats compared to ZL rats, however, GLUT4 failed to reach significance $(\mathrm{p}=0.09$, Figure $3 \mathrm{E}, \mathrm{F}$ and $3 \mathrm{H})$. Further, pyruvate dehydrogenase kinase 4 (PDK4) protein expression remained unchanged between the groups (Figure 3G).

\section{Discussion}

Using echocardiography and ${ }^{18}$ FDG PET, the main finding of this study was that diabetic cardiomyopathy in an experimental model of diabetes is characterised by RV and LV alterations. In particular, changes in RV function were associated with systemic insulin sensitivity and reduced RV insulin-stimulated glucose utilisation. The alterations in RV and LV insulin-stimulated glucose metabolism and systolic function may be attributed to ventricular interdependence, but also indicate that diabetes similarly affects both ventricles.

Impaired RV systolic function as reported in the present study is in agreement with data from Mittal [5] and Movahed et al. [8] who reported comparable results in T2DM patients. Others showed deterioration of RV diastolic function in these patients, whereas the association with RV systolic dysfunction was undecided ambivalent $[6,7,15]$. In addition, the metabolic alterations in the RV as found in the present study are consistent with previously reported findings in ${ }^{18}$ FDG PET studies of the LV in ZDF rats $[9,16]$.

A restriction to glucose utilisation in the diabetic heart is the slow rate of glucose transport [17]. In line with previous reports of the $\operatorname{LV}[9,18,19]$, decreased GLUT4 expression was found, which is compatible with the in vivo change in RV insulin-stimulated glucose metabolism. Impaired insulin signaling is associated with alterations in glucose metabolism. Impaired systemic and RV insulin sensitivity was demonstrated by hyperinsulinaemic euglycaemic clamp and an impaired ability of insulin to phosphorylate Akt and GSK3 $\beta$. Overall these molecular analyses are in line with the molecular changes found in the LV [9], suggesting the uniform effect of diabetes on the heart. Finally, an association was found between RV and LV changes with respect to systolic function and glucose metabolism. 
A

Right ventricle

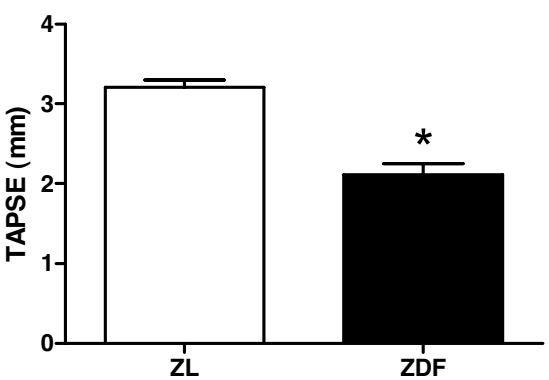

C

Right ventricle

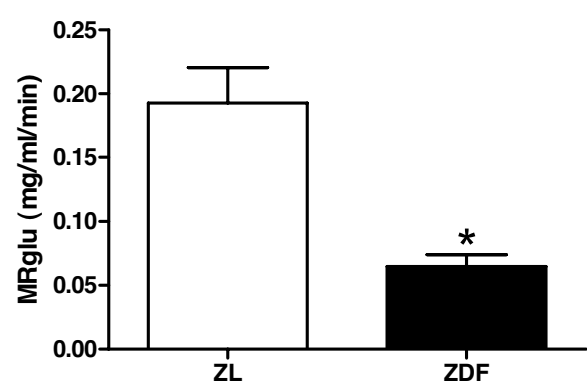

$\mathbf{E}$

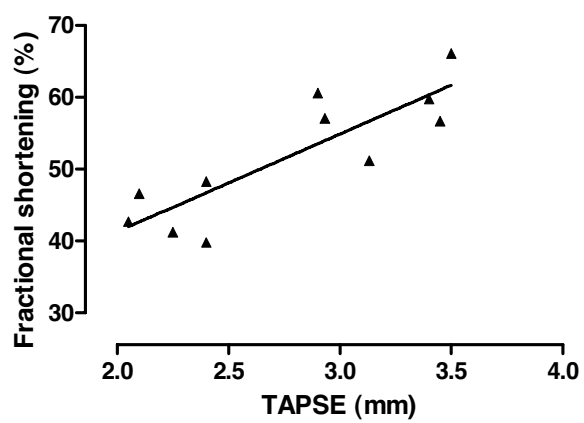

B

Left ventricle

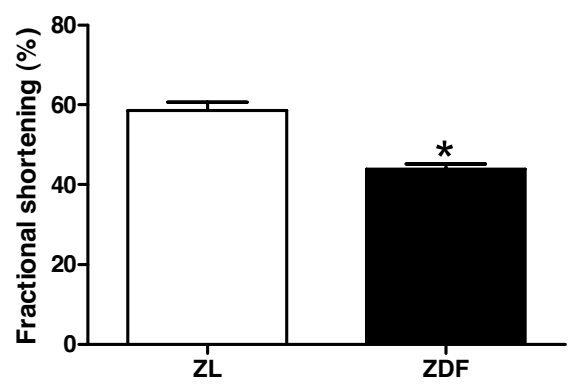

D

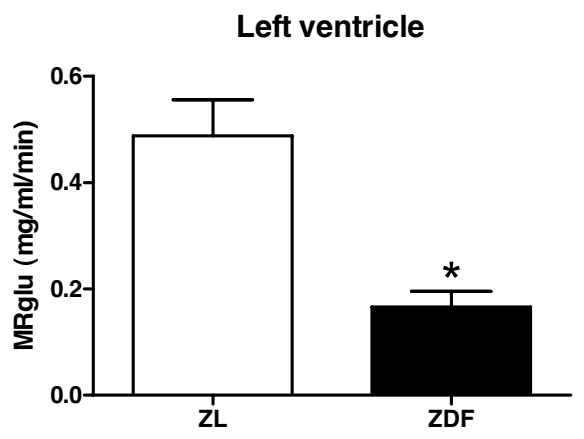

F

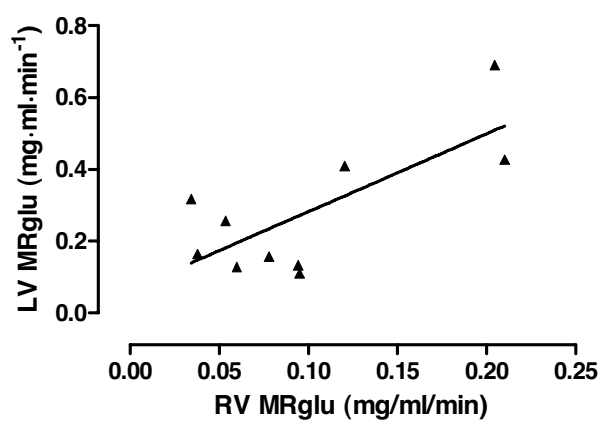

Figure 1 In vivo alterations in right and left ventricular glucose metabolism and function. Relative change in systolic function of RV (tricuspid annulus systolic plane excursion; TAPSE) (A) and LV (fractional shortening) (B) and metabolic rate of glucose utilisation (MRglu) measured under hyperinsulinaemic euglycemic clamp conditions for RV (C) and LV (D) of ZL rats (white bars) and ZDF rats (black bars). Data are expressed as mean \pm SEM, $n=4-9$, ${ }^{*} p<0.05$ vs. ZL rats. Relationship of RV systolic function (TAPSE) with LV systolic function (fractional shortening) $(\mathbf{E} ; r=0.86, p<0.05, n=11$ ) and RV MRglu with LV MRglu $(\mathbf{F} ; r=0.74, p<0.05, n=10)$.

Remodeling of the ventricles was found to be different despite comparable changes in function and metabolism between the ventricles. Despite the absence of fibrosis in both ventricles, LV remodeling in ZDF rats is indicated by hypertrophy of individual cardiomyocytes in the absence of LV dilatation. In contrast, RV remodeling in ZDF rats is indicated by dilatation of the RV in the absence of hypertrophy. This is in agreement with Kosmala et al. [7] who reported the absence of changes in RV free wall thickness. 
A

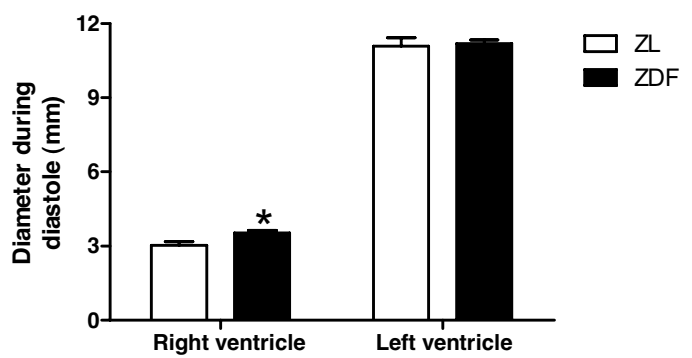

C

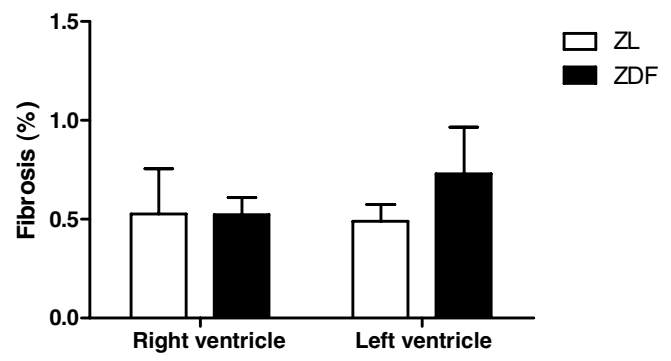

B

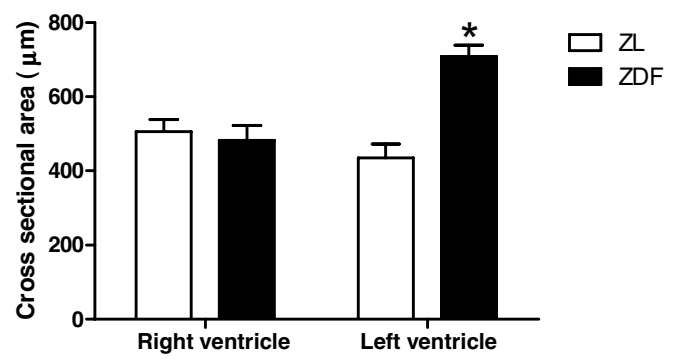

Figure 2 Dilatation of the right ventricle and hypertrophy in the left ventricle. Diastolic diameter of RV and LV (A), cross sectional area of individual cardiomyocytes in RV and LV (B) and quantification of fibrosis (C) of ZL rats (white bars) and ZDF rats (black bars). Data are expressed as mean $\pm \mathrm{SEM}, \mathrm{n}=4-9,{ }^{*} \mathrm{p}<0.05$ vs. ZL rats.

Briefly, the following mechanisms can be hypothesized: 1) increased LV filling pressure is translated to the pulmonary circulation, 2) ventricular interdependence and 3) both ventricles are exposed to the metabolic abnormalities characterising T2DM. Measurements on afterload and RV remodeling remained unchanged; we can therefore conclude that there are no differences in pulmonary vascular resistance. Based on the geometry of the heart it is expected that there is ventricular interdependence. However, in a model of diet-induced obesity, impaired LV function in the absence of RV dysfunction was found (data not shown), which suggests that the effect of diabetes on RV function may be of greater importance than previously assumed. In combination with the in vivo and molecular changes found in RV glucose metabolism and insulin signaling, this suggests that diabetes affects both ventricles.

Collectively, diabetes seems to affect RV glucose metabolism and RV systolic function in similar proportions as in the LV, which could be due to ventricular inter- dependence as well as the uniform effect of diabetes on the heart.

\section{Conclusion}

Whereas consequences of diabetes on the heart were previously restricted to the $\mathrm{LV}$, this study showed that LV insulin-stimulated glucose utilisation and LV systolic function are paralleled by significant alterations in RV insulin-stimulated glucose utilisation and RV function in an experimental rat model of diabetes. RV dysfunction is associated with worse outcome in a variety of cardiovascular diseases, including myocardial infarction and heart failure. The negative association of RV function with prognosis has only been scarcely investigated in the diabetic population. However, since diabetic patients are prone to develop diabetic cardiomyopathy and myocardial ischaemia [4], it might be suggested that RV dysfunction plays a central role in cardiac abnormalities in this population. This warrants further research with respect to the role of RV function in diabetic cardiomyopathy and its relation to patient outcome in diabetes. 
A

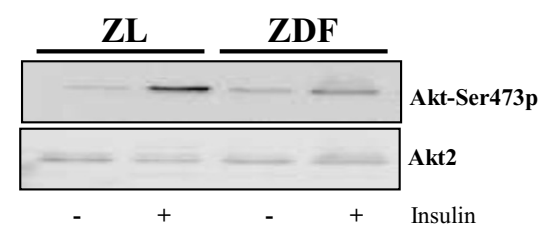

C

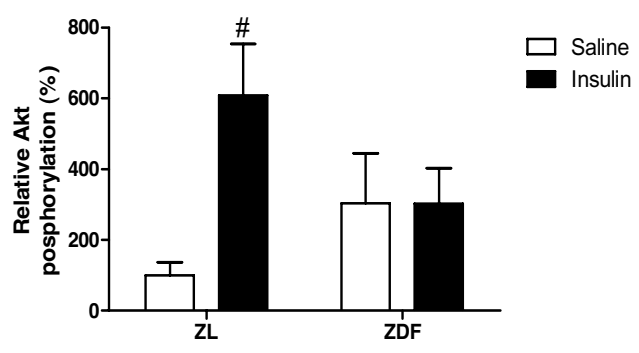

$\mathbf{E}$

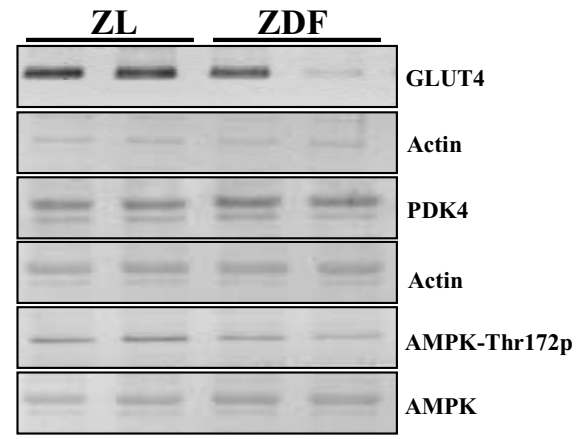

G

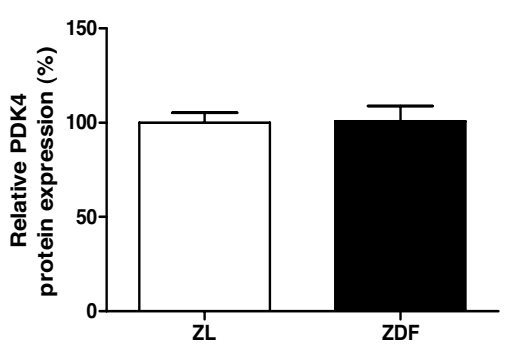

B

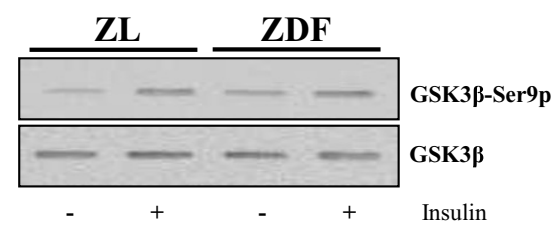

D

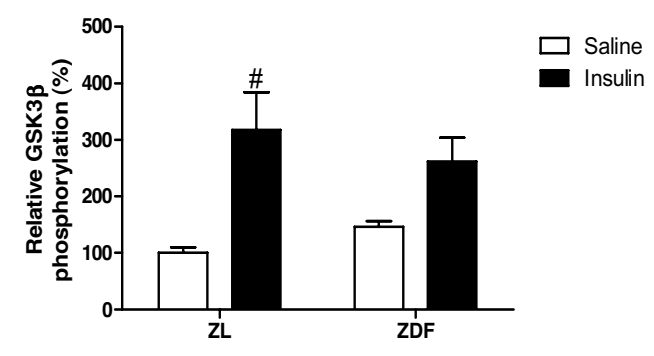

F

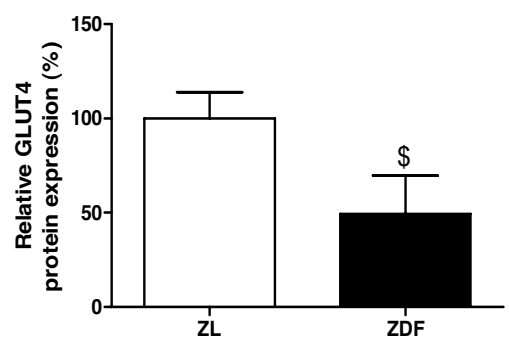

H

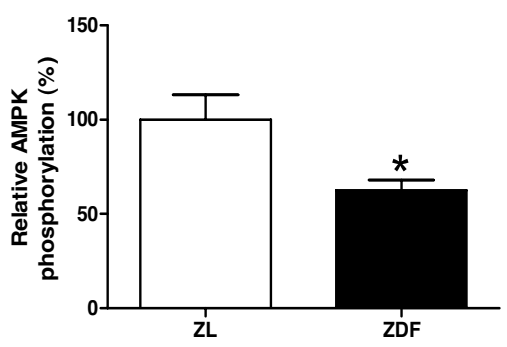

Figure 3 Molecular changes in right ventricular insulin signaling. Immunoblots showing Akt (A) and glycogen synthase kinase (GSK)3 3 (B) and glucose transporter (GLUT)4, pyruvate dehydrogenase kinase (PDK)4 and AMP activated kinase (AMPK) (E). Quantification of immunoblots showing relative phosphorylation of Akt (C) and GSK3 3 (D) after saline (-; open bars) or insulin (+; filled bars) injection in RV. Quantification of immunoblots showing relative protein expression of GLUT4 (F), PDK4 (G) and AMPK phosphorylation (H) in ZL rats (white bars) and ZDF rats (black bars). Data are expressed as mean $\pm \mathrm{SEM}, \mathrm{n}=4,{ }^{*} p<0.05 \mathrm{vs}$. ZL rats, $\$ p=0.09 \mathrm{vs}$. ZL rats, $\# p<0.05$ insulin vs. saline effect. 


\section{Competing interests}

The authors declare that they have no competing interests.

\section{Authors' contributions}

CEvdB participated in performing the study, data analysis, statistics and writing the manuscript. JWAM and RV in part performed the study. MLH contributed in interpretation of data. MCH and ML in part performed data analysis. CFMM in part performed the study and contributed to the design of the study. AAL in part participated in the design of the study and reviewed/edited the manuscript. DMO participated in the design of the study and reviewed/edited the manuscript. MD supervised the study, participated in the design of the study and reviewed/edited the manuscript. CB supervised the study and wrote/ reviewed/edited the manuscript. All authors read and approved the final manuscript.

\section{Acknowledgements}

This work was supported by the Dutch Diabetes Research Foundation (grant 2003.00.029 and 2004.00.052) and EU-COST action BM0602.

\section{Author Details}

'Department of Internal Medicine/Diabetes Centre, VU University Medical Centre, Amsterdam, the Netherlands, 2 Laboratory for Physiology, VU University Medical Centre, Amsterdam, the Netherlands, ${ }^{3}$ Department of Pulmonology, VU University Medical Centre, Amsterdam, the Netherlands, , ${ }^{4}$ Department of Nuclear Medicine \& PET Research, VU University Medical Centre, Amsterdam, the Netherlands, ${ }^{5}$ Department of Anaesthesiology, VU University Medical Centre, Amsterdam, the Netherlands and ${ }^{6} \mathrm{German}$ Diabetes Centre, Institute for Clinical Biochemistry and Pathobiochemistry, Düsseldorf, Germany

Received: 16 April 2010 Accepted: 15 June 2010

Published: 15 June 2010

\section{References}

1. Mehta SR, Eikelboom JW, Natarajan MK, Diaz R, Yi C, Gibbons RJ, Yusuf S: Impact of right ventricular involvement on mortality and morbidity in patients with inferior myocardial infarction. J Am Coll Cardiol 2001, 37:37-43.

2. de Groote P, Millaire A, Foucher-Hossein C, Nugue O, Marchandise X, Ducloux G, Lablanche JM: Right ventricular ejection fraction is an independent predictor of survival in patients with moderate heart failure. J Am Coll Cardiol 1998, 32:948-954.

3. Di Salvo TG, Mathier M, Semigran MJ, Dec GW: Preserved right ventricular ejection fraction predicts exercise capacity and survival in advanced heart failure. J Am Coll Cardiol 1995, 25:1143-1153.

4. Rytter $L$, Troelsen S, Beck-Nielsen H: Prevalence and mortality of acute myocardial infarction in patients with diabetes. Diabetes Care 1985, 8:230-234.

5. Mittal SR: Right ventricular functions in patients with type 2 diabetes below 50 years. J Assoc Physicians India 2007, 55:599-600

6. Kosmala W, Colonna P, Przewlocka-Kosmala M, Mazurek W: Right ventricular dysfunction in asymptomatic diabetic patients. Diabetes Care 2004, 27:2736-2738.

7. Kosmala W, Przewlocka-Kosmala M, Mazurek W: Subclinical right ventricular dysfunction in diabetes mellitus--an ultrasonic strain/strain rate study. Diabet Med 2007, 24:656-663.

8. Movahed MR, Milne N: Presence of biventricular dysfunction in patients with type II diabetes mellitus. Congest Heart Fail 2007, 13:78-80.

9. van den Brom CE, Huisman MC, Vlasblom R, Boontje NM, Duijst S, Lubberink M, Molthoff CF, Lammertsma AA, Van der Velden J, Boer C, et al: Altered myocardial substrate metabolism is associated with myocardial dysfunction in early diabetic cardiomyopathy in rats: studies using positron emission tomography. Cardiovasc Diabetol 2009, 8:39.

10. Fang ZY, Prins JB, Marwick TH: Diabetic cardiomyopathy: evidence, mechanisms, and therapeutic implications. Endocr Rev 2004, 25:543-567.

11. An D, Rodrigues B: Role of changes in cardiac metabolism in development of diabetic cardiomyopathy. Am J Physiol Heart Circ Physiol 2006, 291:H1489-H1506.

12. Ouwens DM, Boer C, Fodor M, de Galan P, Heine RJ, Maassen JA, Diamant $M:$ Cardiac dysfunction induced by high-fat diet is associated with altered myocardial insulin signalling in rats. Diabetologia 2005, 48:1229-1237

13. Handoko ML, Schalij I, Kramer K, Sebkhi A, Postmus PE, van der Laarse WJ, Paulus WJ, Vonk-Noordegraaf A: A refined radio-telemetry technique to monitor right ventricle or pulmonary artery pressures in rats: a useful tool in pulmonary hypertension research. Pflugers Arch 2008, 455:951-959.

14. Ouwens DM, Diamant M, Fodor M, Habets DD, Pelsers MM, El Hasnaoui M, Dang ZC, van den Brom CE, Vlasblom R, Rietdijk A, et al:: Cardiac contractile dysfunction in insulin-resistant rats fed a high-fat diet is associated with elevated CD36-mediated fatty acid uptake and esterification. Diabetologia 2007, 50:1938-1948.

15. Karamitsos TD, Karvounis HI, Dalamanga EG, Papadopoulos CE, Didangellos TP, Karamitsos DT, Parharidis GE, Louridas GE: Early diastolic impairment of diabetic heart: the significance of right ventricle. Int J Cardiol 2007, 114:218-223.

16. Shoghi KI, Gropler RJ, Sharp T, Herrero P, Fettig N, Su Y, Mitra MS, Kovacs A, Finck BN, Welch MJ: Time Course of Alterations in Myocardial Glucose Utilization in the Zucker Diabetic Fatty Rat with Correlation to Gene Expression of Glucose Transporters: A Small-Animal PET Investigation J Nucl Med 2008, 49:1320-1327.

17. Slot JW, Geuze HJ, Gigengack S, James DE, Lienhard GE: Translocation of the glucose transporter GLUT4 in cardiac myocytes of the rat. Proc Nat/ Acad Sci USA 1991, 88:7815-7819.

18. Golfman LS, Wilson CR, Sharma S, Burgmaier M, Young ME, Guthrie PH, Van Arsdall M, Adrogue JV, Brown KK, Swaney JS: Activation of PPARgamma enhances myocardial glucose oxidation and improves contractile function in isolated working hearts of ZDF rats. Am J Physiol Endocrinol Metab 2005, 289:E328-E336.

19. Pelzer T, Jazbutyte V, Arias-Loza PA, Segerer S, Lichtenwald M, Law MP, Schafers M, Ertl G, Neyses L: Pioglitazone reverses down-regulation of cardiac PPARgamma expression in Zucker diabetic fatty rats. Biochem Biophys Res Commun 2005, 329:726-732.

doi: 10.1186/1475-2840-9-25

Cite this article as: van den Brom et al., Diabetic cardiomyopathy in Zucker diabetic fatty rats: the forgotten right ventricle Cardiovascular Diabetology 2010, 9:25

\section{Submit your next manuscript to BioMed Centra and take full advantage of:}

- Convenient online submission

- Thorough peer review

- No space constraints or color figure charges

- Immediate publication on acceptance

- Inclusion in PubMed, CAS, Scopus and Google Scholar

- Research which is freely available for redistribution 